\title{
Efficacy of Dry Needling and Acupuncture in the Treatment of Neck
}

\section{Pain}

\author{
Amnon A. Berger (iD ${ }^{1,}$, , Yao Liu ${ }^{1}$, ${\text { Luke } \text { Mosel }^{2} \text {, Kristin A. Champagne }}^{3}$, Miriam T. Ruoff ${ }^{3}$, Elyse M. \\ Cornett $^{2}$, Alan David Kaye ${ }^{2}$, Farnad Imani (iD ${ }^{4}$, Asadollah Shakeri ${ }^{5,}{ }^{* *}$, Giustino Varrassi (iD ${ }^{6}$, Omar \\ Viswanath (iib) ${ }^{2,7,8,9}$ and Ivan Urits (iD) 2,10 \\ ${ }^{1}$ Department of Anesthesiology, Critical Care, and Pain Medicine, Beth Israel Deaconess Medical Center, Harvard Medical School, Boston, MA, USA \\ ${ }^{2}$ Department of Anesthesiology, Louisiana State University Health Shreveport, Shreveport, LA, USA \\ ${ }^{3}$ School of Medicine, Louisiana State University Health Shreveport, Shreveport, LA, USA \\ ${ }^{4}$ Pain Research Center, Department of Anesthesiology and Pain Medicine, Iran University of Medical Sciences, Tehran, Iran \\ ${ }^{5}$ Department of Anesthesiology and Pain Medicine, Zahedan University of Medical Sciences, Zahedan, Iran \\ ${ }^{6}$ Paolo Procacci Foundation, Roma, Italy \\ ${ }^{7}$ Department of Anesthesiology, College of Medicine-Phoenix, University of Arizona, Phoenix, AZ, USA \\ ${ }^{8}$ Department of Anesthesiology, Creighton University School of Medicine, Omaha, NE, USA \\ ${ }^{9}$ Valley Anesthesiology and Pain Consultants-Envision Physician Services, Phoenix, AZ, USA \\ ${ }^{10}$ Southcoast Physician Group Pain Medicine, Wareham, MA, USA \\ "Corresponding author: Department of Anesthesiology, Critical Care, and Pain Medicine, Beth Israel Deaconess Medical Center, Harvard Medical School, Boston, MA, USA. \\ Email:amnonab@gmail.com \\ ${ }^{* *}$ Corresponding author: Department of Anesthesiology and Pain Medicine, Zahedan University of Medical Sciences, Zahedan, Iran. Email: ashakeri123@yahoo.com
}

Received 2021 February 07; Revised 2021 March 22; Accepted 2021 March 26.

\begin{abstract}
Context: Neck pain is a common phenomenon and affects a large segment of the population. Chronic neck pain, lasting more than 3 months, likely occurs in $10 \%-30 \%$ of patients with acute neck pain and affects up to 288 million cases globally, carrying a significant cost in terms of quality of life, disability, and healthcare dollars. Here we review neck pain background, acupuncture and the evidence that exist to support acupuncture use in chronic neck pain.

Results: Neck pain not only affects quality of life directly, but also contributes to depression, job dissatisfaction and reduced productivity. Unfortunately, neck pain is strongly linked to office and computer work and is likely to continue increasing in prevalence. Traditional treatments, such as analgesics, physical therapy, exercise, and non-invasive therapy bring some relief, and invasive therapy is indicated if anatomical pathologies exist. Acupuncture is a form of integrative medicine, originally described and practiced in traditional Chinese medicine and now expanded to include methods including acupressure, dry needling, and others. Traditionally, it focused on restoring the patient's flow of Qi by puncturing specific points along the meridians. It has previously been shown to be effective in other forms of chronic pain and disability. Clinical trials studying acupuncture for neck pain have shown significant reduction in both pain and associated symptoms. These therapies are reviewed in this text.

Conclusions: Neck pain is a common and significant global problem. Acupuncture, dry needling, and cupping were all shown to be effective in alleviating pain both immediately after treatment, as well as provide long-lasting relief. These treatments are generally safe and inexpensive and should be considered as part of a multimodal approach for the treatment of neck pain. More head-to-head studies will provide better data to support a choice of a specific treatment over another.
\end{abstract}

Keywords: Alternative Medicine, Integrative Medicine, Chinese Medicine, Chronic Pain, Disability

\section{Context}

Acupuncture is the act of placing small needles into defined points on the body. These defined points of the body were characterized by ancient Chinese acupuncture practices which found these points to allow better flow of an energy known as "qi" (1). The theory is that when these points are penetrated by needles, it will open any kind of blockage that is not allowing this energy to flow correctly (2). If an individual has a blockage or an excess of this energy, this is when symptoms will start to appear and thus restoring the balance of the energy is what is thought to relieve the individual of the symptoms their body produces (2). Because of the theory that acupuncture diminishes symptoms, many studies have investigated the effectiveness of acupuncture on things like knee pain, back pain, headaches and other pain conditions, which has resulted in acupuncture being performed in medical offices and insurance companies be- 
ginning to cover the procedures (2). This study has chosen to focus its efforts on the use of acupuncture for neck pain. Neck pain can be classified as non-specific or complicated. Neck pain that is classified as non-specific can be caused by a mechanical issue, such as an acceleration-deceleration movement in a motor vehicle accident, or caused by a postural mechanism (3). Furthermore, complicated neck pain affecting the cervical spine can present with neurological symptoms such as radiculopathy or myelopathy (3). In addition, neck pain can be classified into acute or chronic pain. Acute neck pain can last for weeks to months, but the pain resolves after a short period of time. Chronic neck pain is pain that last longer than $3-6$ months and does not resolve after the acute phase; about $50 \%-85 \%$ of patients with acute neck pain will go on to develop chronic neck pain (4). Neck pain is a highly prevalent disorder; in 2017, there were an estimated 288.7 million global cases (5). The 1-year incidence of neck pain was shown to be $10 \%-21 \%$, with a significant number of cases seen in office or sedentary employees (6). Different approaches exist to the treatment of beck pain, a highly prevalent disorder with significant associated disability. Here we review the evidence of acupuncture in neck pain and the efficacy of such treatments.

\section{Neck Pain}

Neck pain can be characterized many ways but perhaps the best method is by duration. Acute neck pain is characterized typically as lasting less than 6 weeks, subacute less than 3 months and chronic greater than 3 months.

Neck pain has a wide differential diagnosis, but most cases seen are musculoskeletal in nature. It may be difficult to isolate a single cause for neck pain as many times multiple conditions are present. This is further compounded by vague and non-specific symptoms that herald the presentation of degenerative changes (3, 7-9). A pure definition of neck pain that is only perceived from thoracic vertebrae 1 to superior nuchal line. This excludes cervical radiculopathy as this pain is felt in the arm and shoulder. This can be a confusing as cervical radicular pain comes from the neck but is perceived in the shoulder. The innervation of the structure the pain is coming from is more important than the structure itself. This is an important distinction as the approach is different depending on cause and location (10). The global burden of disease (GBD 2005) does include shoulder and upper extremity pain as many studies and reports include these causes as well. Evaluation and diagnosis criteria are centered around excluding causes that would need urgent intervention/evaluation such as trauma, tumors, neurologic infections, and certain rheumatologic conditions. If a patient presents with signs/symptoms that would indicate a serious cause they must be urgently evaluated with appropriate imaging, lab testing, or diagnostic testing to identify a specific cause (11). If a patient is not suspected of having a serious cause, then a detailed history and physical are appropriate. Many cases of neck pain in the absence of trauma or serious symptoms do not require imaging, however plain radiographs are preferred for bony injury/pathology and magnetic resonance imaging is preferred for soft tissue injury $(7,12)$.

Neck pain is a common complaint seen by healthcare personnel, it is estimated that the 1 year incidence of all neck pain is $10.4 \%$ and $21.3 \%$ (6). Mean prevalence is difficult to calculate as prevalence ranges can be as much as $80 \%$. This is due to the prevalence differences in sex, occupation, location and income (6, 13, 14). Per GBD 2005 data neck pain is more prevalent in women, urban settings, and high income countries, with the highest risk age range from 35 - 49 years old $(6,13)$. Remission is difficult to calculate as some sources define remission as a cured state. However the GBD 2005 defines it as an asymptomatic state and found remission rates of $33 \%$ to $65 \%$ (6). While difficult to calculate it is estimated that $10 \%-30 \%$ of patients with acute neck pain will develop chronic neck pain (15). Physical and psychosocial categories can be used to examine the risk factors for neck pain. Job satisfaction and general psychological health is related to neck pain prevalence with depressed mood, high stress and perceived strain being the greatest risk factors. It must be noted that chronic pain can be mood altering itself $(16,17)$. Positioning (most commonly reported) and neck strain levels are a large part of the physical risk factor category but level of physical activity and neck strength, mobility, and endurance also play a part in neck pain $(6,16,17)$. Risk for poor recovery from neck pain include somatization, sleep difficulties female, low level education, and severity of initial neck pain $(6,18$, 19). It is worth noting that many of these risk factors are modifiable.

Pain can be grouped in many ways but perhaps the most common is by clinical significance. This is broken down into serious signs/symptoms/causes and not serious signs/symptoms/causes, common causes and uncommon causes, and valid complaints or not valid complaints with overlap multiple groups. Tumors, neurologic infections, rheumatoid arthritis, ankylosing spondylitis, vasculitis, and fractures are serious but rare (10).

Triage of neck pain is based on history and physical. The Neck Pain Task force recommends neck pain should be split into 4 groups: Grade I, no signs of major pathology and no or little interference with daily activities; grade II, no signs of major pathology, but interference with daily activities; grade III, neurologic signs of nerve compression; grade IV, signs of major pathology. Blunt trauma should al- 
ways be based on NEXUS criteria or Canadian C-spine rule (20).

Current treatment options for neck pain include noninvasive methods including NSAIDS, muscle relaxants, mobilization/manipulation, exercises, electromagnetic therapy/TENS, and acupuncture. NSAIDS and muscle relaxants are quite common however do not carry enough evidence to determine if they are effective and carry the risk of GI upset and drowsiness respectively $(20,21)$. In addition, neck pain in the absence of structural pathology/trauma is recommended to be treated with conservative therapy that is mobilization/manipulation, exercises, acupuncture and electromagnetic therapy/TENS. Manipulation, exercises, and electromagnetic therapy/TENS can cause mild transient increase in pain. These methods usually provide only short term relief $(20,22)$. Opioid-based therapy, whether oral, infusion or transdermal may be effective (23). In trying to avoid opioid use, cannabis has also been gaining recognition in the treatment of chronic pain (24). Infusion therapy may also include options such as ketamine, lidocaine, magnesium, and dexmedetomidine (25-30), while considering possible side effects, such as toxicity, addiction, and psychosocial harm (31, 32). Invasive interventions such as surgery, radiofrequency ablation, surgical, root injections, epidural injections, are not recommended unless patients have neck pain associated with structural disruption or radiculopathy $(20,22$, 33). More novel approaches, such as neurotomy and spinal cord stimulation have been proven to be effective in similar syndromes and may provide relief for neck pain as well (34-40).

The majority of patients with neck pain will recover with conservative treatment despite the many etiologies of neck pain (7). As noted above conservative treatment can provide short term relief to recovery. Regardless many invasive techniques such as radiofrequency ablation and intra-articular steroid injections are quite common for chronic neck pain that is refractory to conservative treatment. However, there are no studies that demonstrate their efficacy. Medial branch block is primarily a diagnostic technique but did demonstrate significant pain relief with repeated injections regardless of whether steroids were included with the local injection $(11,22)$. In the absence of serious pathology, no surgical technique has scientific support. The majority of patient remain significantly impaired post-surgery $(7,33)$. In patient with serious pathology surgery seems superior to non-invasive treatments only in the short term $(3,7)$.

\section{Acupuncture}

Acupuncture is part of traditional Chinese medicine where eliciting pain in one distinct area of the body is found to relieve pain and symptoms in another distinct area of the body (41). Acupuncture is thought to have originated in the fifth century and is a major component of the history of Chinese medicine that has been practiced for over 3,000 years $(42,43)$. In terms of performing the procedure, an acupuncture procedure is performed by placing a number of needles, between 5 and 20, in various defined areas of the body for a period of time (44). The needles can stay in the skin for as long as 20 minutes, and they may be manipulated by the practitioner during the procedure to produce the sensation they are seeking (44). Depending on the symptoms being treated, the initial treatment visit can last up to one hour, and subsequent visits can last half an hour and be as frequent as twice per week (44). A certified acupuncture practitioner will determine the amount of needles, placement of the needles, and frequency of the visits in order to best suit the condition being treated (44). Historically, it is believed that acupuncture was brought to Europe and America during the 18th century and thereafter it has been researched and attempted to be implicated in clinical practice (42). The theory is that acupuncture originated when early human civilization would place warm stones on areas of the body to relieve pain and symptoms they were experiencing (43). Throughout the evolution of acupuncture, pain was relieved by causing pain in another area by evolution methods of stones, bones, bamboo, iron, silver, gold, and now currently stainless steel in the acupuncture that we think of today (41). After acupuncture was introduced to places outside of China and started to gain popularity, many studies have been conducted to determine if there is a benefit in the use of acupuncture in things such as urinary tract infections, neuropathic pain, peripheral neuropathy, hip osteoarthritis, cerebellum balancing, allergic rhinitis, treatment of hypolactation, depression, gynecological conditions, migraine, chronic prostatitis, and post stroke spastic hemiplegia (2, 45-56).

Upon investigation of the specific mechanism of acupuncture, it was determined that traditional Chinese culture proposes that the mechanism of action of acupuncture is centered around the theory that the body has meridians, which are channels connecting each body part to each other, and collaterals, that interconnect the channels between each other (41). The theory is that blood and qi are being transported through these meridians and channels and this transportation must be equal to regulate and keep the body working properly (41). Qi is defined as an energy that is vital in promoting regulation of every function of the body and the goal is to have qi energy flow- 
ing at the right amount, without any obstructions or blockages of the flow (41). When qi energy is not flowing equally, either in excess or lacking, or is found to be blocked, this is when symptoms and pain begin to develop (41). It is through these defined channels and collaterals that distinct acupuncture needle locations have been defined to help reduce pain in a certain body part, to provide the most benefit in equalizing the qi energy when being punctured (41). Aside from the traditional theory of the mechanism, studies have been performed to determine the mechanism of pain relief and one study concluded that the pain relief was not from the qi energy theory, but it was from neuroelectric stimulation causing neuropeptide gene expression (57). Other studies have shown that when the acupuncture needle penetrates afferent nerves, this may cause the release of opioids endogenously that improve pain (58). In addition, studies conducted using functional MRI have concluded that acupuncture effects are linked to specific regions of the brain and their placement impacts important brain structures and function (59). Different methods for acupuncture have been tested; clinical trials have shown efficacy in many fields, including chronic back pain, headache, and recovery time from general anesthesia (56, 60-64).

Once acupuncture is put into practice to achieve the desired mechanism, other things to consider are side effects from the procedure. In a study that investigated retrospective studies, case reports, and surveys of medical providers, they concluded the following to be the most common adverse effects of acupuncture: bruising, needle site bleeding, diarrhea, increased pain, nausea, vomiting, fainting, psychiatric disturbances, headaches, sweating, dizziness, and aggravation of symptoms (65). The same study compiled a list of rare complications which include: pneumothorax, spinal cord injury, hepatitis B, septicemia, injured organs, convulsions, and argyria (65). More specifically, a systemic review on acupuncture safety that looked at nine different studies found that the most common side effects were: needle pain, tiredness, and bleeding (65). Contraindications to acupuncture are in patients with automatic implantable cardioverter defibrillator and in patients who have a history of psychosis or delusions (2). In addition, it is reported that patients who are pregnant or receiving anticoagulation therapy are not completely contraindicated from acupuncture treatment, but they can be at risk for complications (2). Furthermore, in a study investigating the safety of acupuncture use in the pediatric population, they concluded that there is a 1.55 risk of adverse events in 100 acupuncture treatments, which demonstrated that there is a low risk for adverse events in pediatrics (66). Because acupuncture is a procedure that can produce potentially adverse side effects, the FDA has a close regulation on the needles used to perform acupuncture to ensure the safety and sterility of the procedure. The acupuncture needles are currently listed under class II medical devices with the FDA and are grouped in the category of complementary and alternative medicine items (67).

\section{Acupuncture and Needling for Neck Pain}

\subsection{Dry Cupping Studies}

A randomized controlled trial (RCT) pilot study (NCT01289964) investigated dry cupping as a treatment for chronic neck pain (68). Fifty patients aged 18 - 75 with chronic non-specific neck pain were randomized to receive treatment versus "wait list". Pain at rest (PR), pain related to movement (PM), neck disability index (NDI), and quality of life (SF-36) were assessed. The study also included sensory tests: Vibration-detection threshold (VDT), mechanical-detection threshold (MDT), and pressure-pain threshold (PPT). The treatment group received five dry cupping treatments over a two-week period, with no treatment in the control group. Common side effects were short-lived and self-terminated, including pain in tingling in treated areas and upper limbs, headache, and tiredness. One participant discontinued the treatment due to temporarily worsened symptoms. The treatment group reported significantly less pain after dry cupping treatment than the waiting-list group (PR: $\Delta-22.5 \mathrm{~mm}, \mathrm{P}$ $=0.00002 ;$ PM: $\Delta-17.8 \mathrm{~mm}, \mathrm{p}=0.01)$. Analysis of the pain diaries (PD) demonstrated a gradual decrease in neck pain for the treatment group. The pain diaries also showed that after the fifth session, there was a significant difference in pain between the treatment and waiting-list group $(\Delta-1.1$, $\mathrm{P}=0.001)$. The SF-36 subscales for bodily pain $(\Delta 13.8, \mathrm{P}$ $=0.006)$ and vitality $(\Delta 10.2, \mathrm{P}=0.006)$ demonstrated significant differences. There were significant differences between the two groups regarding PPT at pain-related and control areas (all $\mathrm{P}<0.05$ ), but not for MDT or VDT. The study concluded that five dry cupping treatment sessions appeared efficacious in alleviating chronic non-specific neck pain. A significant decrease in mechanical pain sensitivity in the treatment group suggests changed functional pain processing (68).

Another RCT analyzed cupping in chronic neck and shoulder pain (NSP) and measured changes in skin surface temperature (SST) (69). This study randomized 62 participants into a cupping or control group. The cupping group received five cupping therapies at three acupuncture points in the neck/shoulder areas, SI 15, GB 21, and LI 15, for 10 minutes on each side of the body. The control group rested for 20 minutes. SST was measured at 5-minutes interval in the treated points, and blood pressure (BP) was 
measured before and after intervention. Pain was scored using a visual analog scale (VAS), and a Likert scale measured the subjective experience of pain intensity. The study found a significant increase in SST of about $2^{\circ} \mathrm{C}$ in the treatment group, as well as a significant decrease in BP (from $117.7 \pm 2.9 \mathrm{mmHg}$ to $111.8 \pm 2.3 \mathrm{mmHg}$ ), however, there was no significant change in BP from the control group. Pain was significantly reduced in the treatment group, with average VAS decrease of 6.1 points (versus only 0.2 in the control group) for neck pain and 5.9 (versus 0.6 in controls) for shoulder pain (69).

ChiCTR800014723, an RTC, examined the effectiveness of ischemic compression therapy, dry cupping, and the combination of the two therapies in treating trigger points (TPs) associated with neck pain. The study randomized 24 patients to either treatment, or both and measured PPT, neck ROM and NDI pre-treatment and at four weeks post-treatment. All three groups demonstrated significant improvement in NDI, PPT, and neck ROM in comparison to the pre-treatment results. The study did not find a major difference between ischemic compression and dry cupping. The combination of the two treatment methods demonstrated more rapid and greater improvement (70).

A review by Trofa et al. (71) reviewed the above mentioned works and others. Though relatively safe, some studies reported adverse events of post-inflammatory hyperpigmentation, anemia requiring transfusion, formation of abscess, keloids, burns, blisters, body ache, skin laceration, itching, and pain at the site of cupping (71).

\subsection{Dry Needling Studies}

A prospective, nonrandomized study investigated the pain-reducing effects of dry needling on myofascial trigger points (MTrP). Participants experienced shoulder or neck pain for more than three months and had active myofascial trigger points. Each participant received three dry needling treatment sessions weekly. The study's primary outcome measurements included pain evaluations taken at baseline and after treatment using the VAS, the Brief Pain Inventory, and the status of the myofascial trigger point rated as either active, latent, or resolved. Of 52 participants, 41 experienced a trigger point status change from active to latent or resolved, and 11 reported no change. The reduction in all pain scores was significant $(\mathrm{P}<0.001)$. Secondary outcome measurements involved a profile of mood states, Oswestry disability index (ODI), short form 36 (SF36) scores, and cervical ROM. PPT did not significantly change in either unilateral or bilateral MTrPs; physical functioning subscale scores increased significantly, as well as ODI scores, but not VAS scores. There was also improvement in cervical ROM. The study concluded that dry needling significantly reduced pain and changed trigger point status.
The reduced pain was associated with an improvement in function, disability, and mood (72).

A randomized, single-blinded clinical trial (ISRCTN22726482) examined the effectiveness of deep dry needling (DDN) of MTrPs as a treatment for chronic nonspecific neck pain (NSNP). 128 subjects with NSNP and active MTrPs in cervical muscles were randomized to either the DDN group (DDN-plus passive stretching) or the control group (only passive stretching). Each group was provided with four treatments over two weeks and followed up six months later. Some participants experienced soreness and localized bleeding at the site of needle insertion lasting $\leq 1$ week. Significant reductions in PPT, Pain intensity, ROM, strength and perceived disability (73).

A RCT (NCT02301468) examined the short- and longterm treatment effects of dry needling (DN) and manual pressure (MP) for myofascial pain in women. 42 female patients were randomized to receive four treatments of either DN or MP. One participant in the MP group and three in the DN group dropped out. Some soreness after dry needling treatment was reported. The study did not find significant differences between the two groups in PPT, muscle characteristics pre-and post-treatment, the neck disability index (NDI), and pain numeric rating scale (NRS). However, three months post-treatment, both groups experienced significant improvement in NDI and NRS, as well as PPT, muscle elasticity and stiffness (74).

\subsection{Acupuncture Studies}

A systematic review of 265 RCTs and 5 non-RCTs regarding complementary and alternative medicine (CAM) therapies analyzed the efficacy, harm, and cost-effectiveness of acupuncture, massage, spinal manipulation, and mobilization treating pain of the back, neck, and/or thoracic region. The most common adverse events associated with acupuncture included minor bleeding, bruising, soreness, pain at the needling site, lightheadedness, dizziness, and headache. The percentage of participants having had an adverse event was no different than conventional care or TENS groups. In treating chronic non-specific low back pain, acupuncture significantly reduced pain intensity compared to placebo, but only right after treatment. (VAS: -0.59, 95 percent CI:-0.93, -0.25). The review did not find a difference between acupuncture and placebo regarding intake of pain medication, overall improvement in chronic non-specific low back pain, or post-treatment disability. Regarding decreasing chronic non-specific neck pain immediately post-treatment, acupuncture and shamacupuncture did not differ (VAS: $0.24,95$ percent CI: -1.20 , 0.73). Compared to no treatment, acupuncture improved pain intensity (VAS: -1.19, 95 percent CI: 95 percent CI: -2.17, -0.21), disability (pain disability index; PDI), functioning 
(Hannover Functional Ability questionnaire-HFAQ), wellbeing (SF-36), and range of mobility (extension, flexion), immediately post-treatment. Overall, the studies that involved sham-acupuncture more likely had statistically insignificant results versus studies that involved placebos such as laser therapy or medications. Studies produced less consistent results when compared to other treatments such as mobilization. When compared to conventional treatment or no treatment, acupuncture was more economical for treating chronic back pain (75).

A systematic review and meta-analysis examined fourteen RCTs of acupuncture for neck pain. The review included nine meta-analyses, seven of which demonstrated positive. The meta-analysis of short-term pain reduction (continuous data), the primary outcome, showed acupuncture more effective in treating neck pain compared to the control. The analysis also showed that acupuncture was better for pain relief than sham acupuncture. Other positive findings included improvement in neck ROM, cervical radiculopathy, and pain control (both versus sham or no treatment) (76).

This article outlines the design and rationale of a randomized, double-blind, controlled trial (code: ChiCTR-TRC12002206) that intends to examine the safety and efficacy of acupuncture compared to sham acupuncture in treating neck pain caused by cervical spondylosis. The study's design is to randomize 456 recruited participants into either active acupuncture or sham acupuncture group. Treatments will occur five times/week for a total of two weeks. The Northwick Park Neck Pain questionnaire (NPQ) scale, short-form 36 (SF-36) scale, and McGill pain scale, demographics, adverse events, and neck physiological function will be assessed at baseline and intervention for both weeks. Follow-ups will occur at four, eight, and twelve weeks post-intervention. This article was published in 2013, and the study's published outcome is not available per the Chinese Clinical Trial Registry (77).

A study examined acupuncture efficacy with seven acupoint-penetrating needles on sixty patients with cervical spondylosis. The patients were randomized into either group A, which received acupuncture with seven acupointpenetrating needles plus traction or group $\mathrm{B}$, which received acupuncture of non-relevant acupuncture points plus traction. The study compared the two groups based on efficacy and score changes for the Visual Analogue scale (VAS), neck disability index (NDI), and the Pittsburgh sleep quality index (PSQI). Group A demonstrated a significantly higher total effective rate $(90.0 \%)$ compared to group B $(76.6 \%)(\mathrm{P}<0.05)$. The post-treatment scores for VAS, NDI, and PSQI were significantly lower for both groups than the pre-treatment scores $(\mathrm{P}<0.05)$. Group A demonstrated significantly lower NDI and PSQI scores post-treatment than group B $(\mathrm{P}<0.05)$. The study's findings concluded that in patients with cervical spondylosis, acupuncture with seven acupoint-penetrating needles plus traction had greater efficacy, pain reduction, and better quality of sleep versus acupuncture of non-relevant acupuncture points (78).

A study analyzed fast acupuncture versus retaining acupuncture in the treatment of the cervical type of cervical spondylosis. The study randomized 60 participants into either the fast acupuncture group or the retaining needle group. For the fast acupuncture group, the needles were removed after the arrival of qi. For the retaining needle group, the needles remained in place for 30 minutes. The study used the NPQ and short-form McGill pain questionnaire (SF-MPQ) [pain rating index (PRI), Visual Analogue scale (VAS), and present pain intensity (PPI)] to measure outcomes before and after treatment. For both groups, the NPQ score decreased after treatment (both P $<0.01$ ), and the fast acupuncture group had greater improvement $(\mathrm{P}<0.01)$. For both groups, all components of the SF-MPQ were lower compared to the pre-treatment scores (all $\mathrm{P}<0.01$ ). The fast acupuncture group had better PRI sensation and PRI total score (both $\mathrm{P}<0.05$ ). Posttreatment, the PRI feeling score, VAS score, and PPI scores were not significantly different between the two groups (all P > 0.05). With the fast acupuncture group's total effective rate being $83.3 \%$ (25/30), there was not a significant difference from the $76.7 \%$ (23/30) of the retaining needle group $(\mathrm{P}>0.05)$. The study concluded that both fast and retaining needle acupuncture were efficacious in improving the cervical type of cervical spondylosis symptoms. The study found fast acupuncture to be better than retaining needle acupuncture [This article was originally written in Chinese; only the abstract was available in English] (79).

A randomized controlled trial (code: ChiCTR-TRC14004932) investigated the efficacy of abdominal acupuncture to treat neck pain. One hundred fifty-four neck pain patients were randomized to either receive abdominal acupuncture (group A) or non-penetrating sham abdominal acupuncture (group S) and received three treatments/week for two weeks. The Northwick Park Neck Pain questionnaire (NPQ) assessed the primary outcome, mean improvement in neck pain disability. Neck pain intensity and health-related quality-of-life measures were secondary outcome measurements. The study analyzed outcomes at baseline, two weeks, and six weeks after the baseline assessment. An additional follow-up meeting occurs after fourteen weeks from the baseline assessment for the participants who received abdominal acupuncture. In the abdominal acupuncture group, 11 participants developed bruises at the needle insertion site one time each. Neither group reported adverse side effects. The abdominal 
acupuncture group demonstrated greater improvement compared to the sham acupuncture group in NPQ scores at two weeks (intergroup mean differences, -5.75 ; $95 \%$ confidence interval [CI], -9.48 to $-2.03 ; \mathrm{P}=0.008$ ) and at six weeks (intergroup mean difference, $-8.65 ; 95 \% \mathrm{CI},-12.13$ to $-5.16 ; \mathrm{P}<0.001)$. At 14 weeks, the abdominal acupuncture group had significantly greater improvement in NPQ scores compared to baseline. The abdominal acupuncture group had significantly greater improvement than the sham acupuncture group regarding the intensity of neck pain and health-associated quality of life measurements. The findings of the study advocated for the efficacy of abdominal acupuncture in treating neck pain (80).

A randomized, blinded crossover study examined alterations in the myoelectric activity of the upper trapezius and non-specific neck pain after one acupuncture session. The study included fifteen subjects with neck pain (neck pain group, NPG) and fifteen healthy subjects (control group, HPG). Within each group, participants were randomized to either receive one session of acupuncture or sham acupuncture treatment. The acupuncture targeted acupoints of triple energizer 5 (TE-5) and large intestine 11 triple energizer 5 (LI-11 TE-5). The primary outcome was the result of electromyography (EMG) of the upper trapezius muscle. Before and following acupuncture treatment, the EMG signal was recorded during step contractions involving four shoulder elevation force levels (15\%, $20 \%, 24 \%$, and $30 \%$ maximum voluntary contraction). The study demonstrated significant decreases in amplitude of EMG after acupuncture treatment in both the neck pain group $\left(\mathrm{F}_{1,112}=26.82 ; \mathrm{P}<0.0001\right)$ and the control group $\left(\mathrm{F}_{1,112}=21.69 ; \mathrm{P}<0.0001\right)$. For the NPG, the Numeric Rating Scale score (NRS) $\left(\mathrm{F}_{1,28}=51.61 ; \mathrm{P}<0.0001\right)$ and pain area $\left(\mathrm{F}_{1,2}=32.03 ; \mathrm{P}<0.0001\right)$ demonstrated significant effects post-treatment for both acupuncture and sham. The study found no difference between acupuncture and sham acupuncture treatment for NRS score (NPG: $\mathrm{F}_{1,28}=0.95$; $\mathrm{P}$ $=0.33$ ), pain area (NPG: $F_{1,28}=1.97 ; P=0.17$ ), or EMG amplitude (NPG: $\mathrm{F}_{1,112}=0.47 ; \mathrm{P}=0.49 ;$ HPG: $\mathrm{F}_{1,112}=0.75 ; \mathrm{P}=$ 0.38 ). No adverse effects were reported after acupuncture treatment. The study concluded that acupuncture at TE- 5 and LI-11 TE-5 acupoints, or close to these points, helped improve neck pain. For both the NPG and control group, EMG demonstrated decreased hyperactivity of the upper trapezius muscle and increased resistance to muscle fatigue (81).

A review analyzed 25 RCTs regarding noninvasive nonpharmacologic treatments for chronic neck pain. Combination exercise and low-level laser therapy both improved function and pain to a certain degree. Acupuncture provided some functional improvement but did not reduce pain when compared to sham acupuncture. The Alexander technique, a mind-body practice, also demonstrated some improvement in function. Massage did not improve function, and physical therapist-led relaxation techniques did not improve function or pain. All of the above received a strength of recommendation (SOR) of B due to inconsistent or limited-quality evidence that is patient-oriented. The review concluded that physicians should discuss these nonpharmacologic options with patients and consider including combination exercise, low-level laser therapy, acupuncture, and the Alexander technique to treat chronic neck pain (82).

A systemic review and meta-analysis of sixteen RCTs examined the safety and efficacy of acupuncture and electroacupuncture in treating chronic neck pain. The review included nine acupuncture studies and seven electroacupuncture studies. The studies included in the review had treatment groups with or without active control and control groups that received typical intervention options, medication or physical therapy. The review examined the results of disability, quality of life, intensity, and adverse effects. Of note, four trials included in the review had an independent evaluator carry out allocation concealment. Another trial used sealed envelopes. Therefore, the bias risk was considered low for these trials. The remaining eleven studies did not specify allocation concealment or evaluation of bias. There was not a significant difference in pain between the acupuncture group and the active control group (SMD 0.24, 95\% CI:-0.27-0.75), disability (SMD 0.51, 95\% CI: -0.01 - 1.02), or quality of life (SMD -0.37, 95\% CI: -1.09 - 0.35). Acupuncture added into the control group demonstrated greater pain relief in the studies with unclear allocation concealment (SMD -1.78, 95\% CI: -2.08 - 1.48). However, when acupuncture was added into the control group in studies with good allocation concealment there was no significant pain relief (SMD - 0.07, 95\% CI: 0.26 - 0.12). The electroacupuncture versus the control or electroacupuncture plus the active control demonstrated significant pain relief. However, the findings had a low level of evidence. The review noted that no adverse events were reported. The review concluded that acupuncture and conventional therapy demonstrated similar effects regarding pain relief and disability. Pain relief was better when acupuncture was added onto conventional therapy, and electroacupuncture lessened pain to a greater degree. The review stated that additional studies are needed as forming a conclusion was challenging due to the risk of bias and unreliability of the studies (83).

A randomized clinical trial (code: IRCT20100127003217N12) investigated electroacupuncture and biofeedback along with conventional therapy in treating cervical myofascial pain syndrome (MPS). Fifty participants were recruited and randomized into two groups. The 25 participants in the electroacupuncture 
group completed the study. Twenty-five participants in the biofeedback group started the study, but only 23 completed the study. Both groups received their respective interventions twice/week with six sessions in total. All participants received $7.5 \mathrm{mg}$ of meloxicam once daily and taught exercises for shoulder and neck muscles. The study assessed VAS for the severity of pain, NDI, ROM, and PPT at pre-treatment, three weeks, and twelve weeks posttreatment. The study set the primary outcome, measured through NDI, as a $20 \%$ reduction in dysfunction and neck pain at three months compared to the baseline measurement. The electroacupuncture and biofeedback group demonstrated significant improvement in all parameters except for the PPT of the paravertebral muscles and lower trapezius. The acupuncture group reached the primary outcome to a greater extend compared to the biofeedback group: 20 (80.0\%) vs 10 (40.0\%); rate ratio $=2$ with $95 \% \mathrm{CI}$, 1.19 - 3.36; number needed to treat $(\mathrm{NNT})=2.5$ with $95 \% \mathrm{CI}$, 1.54 - 6.58. Acupuncture showed greater clinical benefit compared to biofeedback as per the values of NDI, VAS, extension and left lateral-bending ROM, and PPT on the left upper trapezius after the last treatment session until the 3-month follow-up ( $\mathrm{P}<0.05)$. The study found both electroacupuncture and biofeedback therapies coupled with medication and exercises to decrease pain, increase PPT and cervical ROM, and decrease functional disability in patients with myofascial neck pain. Intergroup differences demonstrated that for some parameters, electroacupuncture was better than biofeedback. The study concluded that electroacupuncture appeared to be superior in treating cervical MPS (84).

An RCT (KCT0002320) examined the safety and efficacy of thread-embedding acupuncture (TEA) using polydioxanone along with conventional care for patients with chronic non-specific neck pain (CNP) versus treatment with only conventional care. A total of $106 \mathrm{CNP}$ participants were randomized to either the TEA plus usual care (TU) group or the usual care (UC) group. TEA treatments occurred once weekly for a total of four weeks. The study defined typical care for treating neck pain to include physical therapy, massage, spinal manipulation, and analgesics. Typical care was provided as needed for patients in both groups. The study's primary outcome measurement was the mean Neck Pain and Disability scale (NPDS) score. The study's secondary outcome measurements were pressure pain threshold (PPT), Hospital Anxiety and Depression scale (HADS), clinically important difference (CID), EuroQol-5 dimension (EQ-5D), and patient global impression of change (PGIC). Assessments occurred at baseline, 3 , 5, and 9 weeks. In the TU group, 12 participants reported adverse events associated with TEA of neck stiffness, bruising, skin flare, pruritus, and irritation due to the thread.
NPDS scores significantly improved in the TU group compared with the UC group (adjusted group difference, week 5: 13.74 [95\% CI: 7.57 - 19.90]; P < 0.0001 and week 9: 17.46 [95\% CI:11.15 - 23.76]; P < 0.0001). For the TU group at weeks 5 and 9, the fraction of patients with a lower NPDS score of $¥ 11.5$ points (minimal CID) was significantly higher than the UC group. For both groups at weeks 5 and 9, the study showed significant differences on the HADS, EQ-5D, and PGIC, but not for the PPTs at the three measured sites, the insertion area of the levator scapulae muscle, GB21 of the upper trapezius, and the space extending $1.5 \mathrm{~cm}$ out from the sixth cervical vertebra. The study's findings indicated TEA, along with conventional care, to be safe and effective in treating CNP (85).

A systemic review and meta-analysis (CRD42016042956) examined six RCTs regarding the efficacy of acupuncture in treating localized unremitting myofascial pain of the head and neck. The study's primary outcome measurement was the mean pain severity score (VAS) for acupuncture versus sham-needling or no intervention groups. No serious adverse events were reported. There was a decrease in VAS scores for acupuncture groups compared to sham needling/no intervention groups in all six studies. The meta-analysis included only four of the RCTs and showed acupuncture's pain intensity score 19.04 points less than sham-needling/no intervention's score (95\% CI: -29.13 to -8.95 ). The findings propose that acupuncture may be safe and efficacious for decreasing unremitting myofascial pain of the head and neck. Additional studies with a more standardized design are needed to provide further compelling evidence (86).

Several RCTs are ongoing or in the recruitment phases, mostly in China. These focus more on head-to-head trials, that are missing from the landscape and would better allow to correctly choose the type of intervention for patients. ChiCTR-IOR-15006886 is a Chinese RCT that aims to investigate the effectiveness of acupuncture in treating chronic neck pain versus sham acupuncture. The study's design is to randomize 175 recruited patients into five separate groups: a traditional acupuncture group (group A), a shallow-puncture group (group B), a non-acupoint acupuncture group (group C), a non-acupoint shallowpuncture group (group D), and a sham-puncture group (group E) (87). Other planned trials will evaluate acupuncture for spondylosis (88), compare high and low sensitivity acupuncture to sham (89), and collect biometric data comparing healthy volunteers and participants with NSNP (90). 


\section{Conclusions}

Chronic neck pain is neck pain lasting 3 - 6 months, pain that does not resolve after an acute phase. This can, unfortunately, happen in up to $85 \%$ of patients suffering from acute neck pain, as estimated by some studies, though more conservative numbers likely place this at $10 \%$ - 30\%. This leads to extremely high prevalence, estimated as high as 288 million cases globally in 2017. Most commonly, neck pain is of musculoskeletal sources, and most risk factors are modifiable; though, these risk factors -such as office and computer work-continue to increase in prevalence. Neck pain often leads to depression, reduced satisfaction, and disability.

Traditional treatment for neck pain includes pharmacologic agents, such as NSAIDs, muscle relaxants and prescription analgesics. Mobilization and manipulation, physical exercise and therapy, as well as electric (TENS) and electromagnetic therapy. These conservative treatments are recommended when no anatomical pathology exists; if the latter does occur, more invasive methods, such as intra-articular injections, radiofrequency ablation, medial branch blocks and surgery may be indicated. These invasive methods carry increased risks that must be weighed in light of the specific pathology and patient choice.

Acupuncture involves inserting thin, firm needles into specific points in a patient body in hopes to clear blockages and allow free flow of a person's Qi. It has been used in traditional Chinese medicine for hundreds of years and recently explored through the eyes of Western Medicine in several different conditions of chronic pain and disability. Acupuncture has been expanded to include, beyond the traditional Chinese methods, other methods that include dry needling, heat acupuncture, acupressure and electropuncture. Dry cupping does not involve needling but is also similar in method otherwise. Though the actual method through which acupuncture alleviates pain is not clearly understood, studies show that it likely involved nerve stimulation via tactile skin stimulation leading to the release of neuromodulators into the plasma and CSF, which then lead to pain alleviation.

The above listed evidence shows a plethora of data, most of it supporting the use of acupuncture and other CAM in the treatment of neck pain. Indeed, Table 1 systematically summarizes the available evidence available for the use of acupuncture in chronic neck pain. Acupuncture was shown to have both immediate and long-lasting effect on pain, disability, and related symptoms. It is generally safe and inexpensive. Unfortunately, very few headto-head trials exist, and it is hard to point at a certain intervention as superior to others.

\section{Footnotes}

Authors' Contribution: Study concept and design: AAB, YL, LM, MAC, MTR, EMC, ADK, and IU. Analysis and interpretation of data: AAB, YL, LM, MAC, and MTR. Drafting of the manuscript: AAB, YL, LM, MAC, MTR, EMC, ADK, FI, AS, GV, OV, and IU. Critical revision of the manuscript for important intellectual content: AAB, YL, LM, MAC, MTR, EMC, ADK, FI, AS, GV, OV, and IU. Statistical analysis: AAB, YL, LM, MAC, and MTR.

Conflict of Interests: The authors have no conflicts of interest to disclose.

Funding/Support: No funding was received for the completion of this manuscript.

\section{References}

1. Yang Y, Wang LP, Zhang L, Wang LC, Wei J, Li JJ, et al. Factors contributing to de qi in acupuncture randomized clinical trials. Evid Based Complement Alternat Med. 2013;2013:329392. doi: 10.1155/2013/329392. [PubMed: 23818924]. [PubMed Central: PMC3683432].

2. Hal MV, Dydyk AM, Green MS. StatPearls. Treasure Island (FL): StatPearls Publishing; 2020.

3. Binder AI. Neck pain. BMJ Clin Evid BMJ Publishing Group; 2008.

4. Goode AP, Freburger J, Carey T. Prevalence, practice patterns, and evidence for chronic neck pain. Arthritis Care Res (Hoboken). 2010;62(11):1594-601. doi: 10.1002/acr.20270. [PubMed: 20521306]. [PubMed Central: PMC2974793]

5. Fejer R, Kyvik KO, Hartvigsen J. The prevalence of neck pain in the world population: a systematic critical review of the literature. Eur Spine J. 2006;15(6):834-48. doi: 10.1007/s00586-004-0864-4. [PubMed: 15999284]. [PubMed Central: PMC3489448].

6. Hoy DG, Protani M, De R, Buchbinder R. The epidemiology of neck pain. Best Pract Res Clin Rheumatol. 2010;24(6):783-92. doi: 10.1016/j.berh.2011.01.019. [PubMed: 21665126].

7. Cohen SP. Epidemiology, diagnosis, and treatment of neck pain. Mayo Clin Proc. 2015;90(2):284-99. doi: 10.1016/j.mayocp.2014.09.008. [PubMed: 25659245].

8. Malik KM, Beckerly R, Imani F. Musculoskeletal Disorders a Universal Source of Pain and Disability Misunderstood and Mismanaged: A Critical Analysis Based on the U.S. Model of Care. Anesth Pain Med. 2018;8(6). e85532. doi: 10.5812/aapm.85532. [PubMed: 30775292]. [PubMed Central: PMC6348332].

9. Khan TW, Imani F. The Management of Chronic Pain; Caught Between a Rock and a Hard Place: The Case for a Renewed Focus on Provider, Patient, and Payer Education. Anesth Pain Med. 2017;7(1). e40951. doi: 10.5812/aapm.40951. [PubMed: 28920037]. [PubMed Central: PMC5554427].

10. Bogduk N. The anatomy and pathophysiology of neck pain. Phys Med Rehabil Clin N Am. 2003;22(3):455-72. doi: 10.1016/S10479651(03)00041-X. [PubMed: 12948338]

11. Childs JD, Cleland JA, Elliott JM, Teyhen DS, Wainner RS, Whitman JM, et al. Neck pain: Clinical practice guidelines linked to the International Classification of Functioning, Disability, and Health from the Orthopedic Section of the American Physical Therapy Association. J Orthop Sports Phys Ther. 2008;38(9):A1-A34. doi: 10.2519/jospt.2008.0303. [PubMed:18758050].

12. Corwell BN, Davis NL. The Emergent Evaluation and Treatment of Neck and Back Pain. Emerg Med Clin North Am. 2020;38(1):167-91. doi: 10.1016/j.emc.2019.09.007. [PubMed: 31757249]. 
13. Cote P, van der Velde G, Cassidy JD, Carroll LJ, Hogg-Johnson S, Holm LW, et al. The burden and determinants of neck pain in workers: results of the Bone and Joint Decade 2000-2010 Task Force on Neck Pain and Its Associated Disorders. Spine (Phila Pa 1976). 2008;33(4 Suppl):S60-74. doi: 10.1097/BRS.0b013e3181643ee4. [PubMed: 18204402].

14. Ris I, Juul-Kristensen B, Boyle E, Kongsted A, Manniche C, Sogaard K. Chronic neck pain patients with traumatic or non-traumatic onset: Differences in characteristics. A cross-sectional study. Scand J Pain. 2017;14:1-8. doi: 10.1016/j.sjpain.2016.08.008. [PubMed: 28850421].

15. Bogduk N. The anatomy and pathophysiology of neck pain. Phys Med Rehabil Clin NAm. 2011;22(3):367-82. vii. doi: 10.1016/j.pmr.2011.03.008. [PubMed: 21824580].

16. Shahidi B, Curran-Everett D, Maluf KS. Psychosocial, Physical, and Neurophysiological Risk Factors for Chronic Neck Pain: A Prospective Inception Cohort Study. J Pain. 2015;16(12):1288-99. doi: 10.1016/j.jpain.2015.09.002. [PubMed: 26400680].

17. Kim R, Wiest C, Clark K, Cook C, Horn M. Identifying risk factors for first-episode neck pain: A systematic review. Musculoskelet Sci Pract. 2018;33:77-83. doi:10.1016/j.msksp.2017.11.007. [PubMed: 29197234].

18. Hendriks EJM, Scholten-Peeters GGM, van der Windt D, Neeleman-van der Steen CWM, Oostendorp RAB, Verhagen AP. Prognostic factors for poor recovery in acute whiplash patients. Pain. 2005;114(3):408-16. doi: 10.1016/j.pain.2005.01.006. [PubMed: 15777866].

19. Ris I, Barbero M, Falla D, Larsen MH, Kraft MN, Sogaard K, et al. Pain extent is more strongly associated with disability, psychological factors, and neck muscle function in people with non-traumatic versus traumatic chronic neck pain: a cross sectional study. Eur J Phys Rehabil Med. 2019;55(1):71-8. doi: 10.23736/S1973-9087.18.04977-8. [PubMed: 30156084].

20. Guzman J, Haldeman S, Carroll LJ, Carragee EJ, Hurwitz EL, Peloso P, et al. Clinical practice implications of the Bone and Joint Decade 2000-2010 Task Force on Neck Pain and Its Associated Disorders: from concepts and findings to recommendations. Spine (Phila Pa 1976). 2008;33(4 Suppl):S199-213. doi: 10.1097/BRS.0b013e3181644641. [PubMed: 18204393].

21. Peck J, Urits I, Peoples S, Foster L, Malla A, Berger AA, et al. A Comprehensive Review of Over the Counter Treatment for Chronic Low Back Pain. Pain Ther. 2020. doi: 10.1007/s40122-020-00209-w. [PubMed: 33150555].

22. van Eerd M, Patijn J, Lataster A, Rosenquist RW, van Kleef M, Mekhail N, et al. 5. Cervical facet pain. Pain Pract. 2010;10(2):113-23. doi: 10.1111/j.1533-2500.2009.00346.x. [PubMed: 20415728].

23. Hemati K, Zaman B, Hassani V, Imani F, Dariaie P. Efficacy of fentanyl transdermal patch in the treatment of chronic soft tissue cancer pain. Anesth Pain Med.2015;5(1). e22900. doi:10.5812/aapm.22900. [PubMed: 25789240]. [PubMed Central: PMC4350185].

24. Berger AA, Keefe J, Winnick A, Gilbert E, Eskander JP, Yazdi C, et al. Cannabis and cannabidiol (CBD) for the treatment of fibromyalgia. Best Pract Res Clin Anaesthesiol. 2020;34(3):617-31. doi: 10.1016/j.bpa.2020.08.010. [PubMed:33004171].

25. Imani F, Varrassi G. Ketamine as Adjuvant for Acute Pain Management. Anesth Pain Med. 2019;9(6). e100178. doi: 10.5812/aapm.100178. [PubMed: 32280623]. [PubMed Central: PMC7119219].

26. Urits I, Virgen CG, Alattar H, Jung JW, Berger AA, Kassem H, et al. A Comprehensive Review and Update of the Use of Dexmedetomidine for Regional Blocks. Psychopharmacol Bull. 2020;50(4 Suppl 1):121-41. [PubMed: 33633422]. [PubMed Central: PMC7901136].

27. Berger AA, Urits I, Hasoon J, Kaye AD, Viswanath O, Eskander J. Pain Alleviation and Opioid Weaning in an 80-Year-Old with Chronic Foot Pain Following Injection Therapy with Perineural Dexmedetomidine and Dexamethasone. Surg J (N Y). 2021;7(1):e1-2. doi: 10.1055/s-00401722176. [PubMed: 33437869]. [PubMed Central: PMC7790527].

28. Urits I, Jung JW, Amgalan A, Fortier L, Anya A, Wesp B, et al. Utilization of Magnesium for the Treatment of Chronic Pain. Anesth Pain Med. 2021;11(1):e112348. doi:10.5812/aapm.112348.
29. Tully J, Jung JW, Patel A, Tukan A, Kandula S, Doan A, et al. Utilization of Intravenous Lidocaine Infusion for the Treatment of Refractory Chronic Pain. Anesth Pain Med. 2020;10(6):e112290. doi: 10.5812/aapm.112290.

30. Imani F, Zaman B, De Negri P. Postoperative Pain Management: Role of Dexmedetomidine as an Adjuvant. Anesth Pain Med. 2020;10(6):e112176. doi: 10.5812/aapm.112176.

31. Malik KM, Imani F, Beckerly R, Chovatiya R. Risk of Opioid Use Disorder from Exposure to Opioids in the Perioperative Period: A Systematic Review. Anesth Pain Med. 2020;10(1). e101339. doi: 10.5812/aapm.101339. [PubMed: 32337175]. [PubMed Central: PMC7158240].

32. Urits I, Gress K, Charipova K, Li N, Berger AA, Cornett EM, et al. Cannabis Use and its Association with Psychological Disorders. Psychopharmacol Bull. 2020;50(2):56-67. [PubMed: 32508368]. [PubMed Central: PMC7255842].

33. Carragee EJ, Hurwitz EL, Cheng I, Carroll LJ, Nordin M, Guzman J, et al. Treatment of neck pain: injections and surgical interventions: results of the Bone and Joint Decade 2000-2010 Task Force on Neck Pain and Its Associated Disorders. Spine (Phila Pa 1976). 2008;33(4 Suppl):S15369. doi: 10.1097/BRS.0b013e31816445ea. [PubMed: 18204388].

34. Berger AA, Hasoon J, Urits I, Viswanath O, Gill J. $10 \mathrm{kHz}$ Spinal Cord Stimulation for Combined Alleviation of Post-Laminectomy Syndrome and Chronic Abdominal Pain: A Case Report. J Pain Res. 2020;13:873-5. doi: 10.2147/JPR.S244084. [PubMed: 32431535]. [PubMed Central: PMC7198401].

35. Hasoon J, Berger AA, Urits I, Orhurhu V, Viswanath O, Aner M. Spinal cord stimulation for the treatment of chronic pelvic pain after Tarlov cyst surgery in a 66-year-old woman: A case report. Case Rep Womens Health. 2020;25. e00171. doi: 10.1016/j.crwh.2020.e00171. [PubMed: 31956516]. [PubMed Central: PMC6962650].

36. Berger AA, Urits I, Hasoon J, Gill J, Aner M, Yazdi CA, et al. Improved Pain Control with Combination Spinal Cord Stimulator Therapy Utilizing Sub-perception and Traditional Paresthesia Based Waveforms: A Pilot Study. Anesth Pain Med. 2021;11(1):e113089. doi: 10.5812/aapm.113089.

37. Imani F. Using pulsed radiofrequency for chronic pain. Anesth Pain Med. 2012;1(3):155-6. doi: 10.5812/kowsar.22287523.4047. [PubMed: 24904784]. [PubMed Central: PMC4018683].

38. Hasoon J, Berger AA. Radiofrequency neurotomy for long-term relief of third occipital neuralgia. Saudi J Anaesth. 2020;14(2):266-7. doi: 10.4103/sja.SJA_666_19. [PubMed: 32317894]. [PubMed Central: PMC7164473].

39. Sluijter ME, Imani F. Evolution and mode of action of pulsed radiofrequency. Anesth Pain Med. 2013;2(4):139-41. doi: 10.5812/aapm.10213. [PubMed: 24223349]. [PubMed Central: PMC3821144].

40. Urits I, Schwartz R, Smoots D, Koop L, Veeravelli S, Orhurhu V, et al. Peripheral Neuromodulation for the Management of Headache. Anesth Pain Med. 2020;10(6):e110515. doi:10.5812/aapm.110515.

41. Ifrim Chen F, Antochi AD, Barbilian AG. Acupuncture and the retrospect of its modern research. Rom J Morphol Embryol. 2019;60(2):411-8. [PubMed: 31658313].

42. Zhuang Y, Xing JJ, Li J, Zeng BY, Liang FR. History of acupuncture research. Int Rev Neurobiol. 2013;111:1-23. doi: 10.1016/B978-0-12-4115453.00001-8. [PubMed: 24215915].

43. Wang CC, Zhu R, Tan JY. Nurses and Holistic Modalities: The History of Chinese Medicine and Acupuncture. Holist Nurs Pract. 2019;33(2):90-4. doi: 10.1097/HNP.0000000000000312. [PubMed: 30747777].

44. Mayo Clinic Staff. Acupuncture - Mayo Clinic. 2019. Available from: https://www.mayoclinic.org/tests-procedures/acupuncture/careat-mayo-clinic/pcc-20392770.

45. Qin X, Coyle ME, Yang L, Liang J, Wang K, Guo X, et al. Acupuncture for recurrent urinary tract infection in women: a systematic review and meta-analysis. BJOG. 2020;127(12):1459-68. doi: 10.1111/1471-0528.16315. [PubMed: 32406571]. 
46. Natbony LR, Zhang N. Acupuncture for Migraine: a Review of the Data and Clinical Insights. Curr Pain Headache Rep. 2020;24(7):32. doi: 10.1007/s11916-020-00864-w. [PubMed: 32472196]

47. Li J, Dong L, Yan X, Liu X, Li Y, Yu X, et al. Is Acupuncture Another Good Choice for Physicians in the Treatment of Chronic Prostatitis/Chronic Pelvic Pain Syndrome? Review of the Latest Literature. Pain Res Manag. 2020;2020:5921038. doi: 10.1155/2020/5921038. [PubMed: 32256909]. [PubMed Central: PMC7085851].

48. Fan W, Kuang X, Hu J, Chen X, Yi W, Lu L, et al. Acupuncture therapy for poststroke spastic hemiplegia: A systematic review and metaanalysis of randomized controlled trials. Complement Ther Clin Pract. 2020;40:101176. doi: 10.1016/j.ctcp.2020.101176. [PubMed: 32347210].

49. Ju ZY, Wang K, Cui HS, Yao Y, Liu SM, Zhou J, et al. Acupuncture for neuropathic pain in adults. Cochrane Database Syst Rev. 2017;12. CD012057. doi: 10.1002/14651858.CD012057.pub2. [PubMed: 29197180]. [PubMed Central: PMC6486266].

50. Dimitrova A, Murchison C, Oken B. Acupuncture for the Treatment of Peripheral Neuropathy: A Systematic Review and Meta-Analysis. J Altern Complement Med. 2017;23(3):164-79. doi:10.1089/acm.2016.0155. [PubMed: 28112552]. [PubMed Central: PMC5359694].

51. Manheimer E, Cheng K, Wieland LS, Shen X, Lao L, Guo M, et al. Acupuncture for hip osteoarthritis. Cochrane Database Syst Rev. 2018;5. CD013010. doi: 10.1002/14651858.CD013010. [PubMed: 29729027]. [PubMed Central: PMC5984198]

52. Elkiss M. Expanding Medical Acupuncture to Promote Balance: The Role of Cerebellar Functioning. Med Acupunct. 2020;32(2):66-70 doi: 10.1089/acu.2020.1408. [PubMed: 32351659]. [PubMed Central: PMC7187970].

53. Zhang J, Zhang Y, Huang X, Lan K, Hu L, Chen Y, et al. Different Acupuncture Therapies for Allergic Rhinitis: Overview of Systematic Reviews and Network Meta-Analysis. Evid Based Complement Alternat Med. 2020;2020:8363027. doi: 10.1155/2020/8363027. [PubMed. 32382307]. [PubMed Central: PMC7195651].

54. Li M, Niu J, Yan P, Yao L, He W, Wang M, et al. The effectiveness and safety of acupuncture for depression: An overview of meta-analyses. Complement Ther Med. 2020;50:102202. doi 10.1016/j.ctim.2019.102202. [PubMed: 32444032]

55. Ernst E, Lee MS, Choi TY. Acupuncture in obstetrics and gynecology: an overview of systematic reviews. Am J Chin Med. 2011;39(3):423-31 doi: 10.1142/S0192415X11008920. [PubMed: 21598411].

56. Li YX, Xiao XL, Zhong DL, Luo LJ, Yang H, Zhou J, et al. Effectiveness and Safety of Acupuncture for Migraine: An Overview of Systematic Reviews. Pain Res Manag. 2020;2020:3825617. doi: 10.1155/2020/3825617. [PubMed: 32269669]. [PubMed Central: PMC7125485].

57. Ulett GA, Han J, Han S. Traditional and evidence-based acupuncture: history, mechanisms, and present status. South Med J.1998;91(12):111520. doi: 10.1097/00007611-199812000-00004. [PubMed: 9853722].

58. Vanderploeg K, Yi X. Acupuncture in modern society. J Acupunct Meridian Stud. 2009;2(1):26-33. doi: 10.1016/S2005-2901(09)60012-1. [PubMed: 20633471].

59. Kaptchuk TJ. Acupuncture: theory, efficacy, and practice. Ann Intern Med. 2002;136(5):374-83. doi: 10.7326/0003-4819-136-5-20020305000010. [PubMed: 11874310].

60. Rezvani M, Alebouyeh MR, Imani F, Entezary SR, Mohseni M. Does changes in the electrical resistance of an acupuncture meridian predict pain intensity following orthopedic surgery? Anesth Pain Med. 2013;2(4):178-81. doi: 10.5812/aapm.7254. [PubMed: 24223357]. [PubMed Central: PMC3821137].

61. Urits I, Wang JK, Yancey K, Mousa M, Jung JW, Berger AA, et al. Acupuncture for the Management of Low Back Pain. Curr Pain Headache Rep. 2021;25(1):2. doi:10.1007/s11916-020-00919-y. [PubMed: 33443607].

62. Farahmand S, Shafazand S, Alinia E, Bagheri-Hariri S, Baratloo A. Pain Management Using Acupuncture Method in Migraine Headache Patients; A Single Blinded Randomized Clinical Trial. Anesth Pain Med. 2018;8(6). e81688. doi: 10.5812/aapm.81688. [PubMed: 30666295]. [PubMed Central: PMC6334036]
63. Faiz SHR, Nikoubakht N, Imani F, Ziyaeifard M, Sadegh H, Rahimzadeh P. Comparison of Two Acupuncture Protocols (K1, DU25 or K1, DU26) Efficacy on Recovery Time of Patients After General Anesthesia, a Randomized Control Clinical Trial. Anesth Pain Med. 2019;9(5). e96172. doi: 10.5812/aapm.96172. [PubMed: 31903336]. [PubMed Central: PMC6935292].

64. Berger AA, Liu Y, Jin K, Kaneb A, Welschmeyer A, Cornett EM, et al. Efficacy of Acupuncture in the Treatment of Chronic Abdominal Pain. Anesth Pain Med. 2021;11(2):e113027. doi: 10.5812/aapm.113027.

65. Chung A, Bui L, Mills E. Adverse effects of acupuncture. Which are clinically significant? Can Fam Physician. 2003;49:985-9. [PubMed: 12943357]. [PubMed Central: PMC2214278].

66. Jindal V, Ge A, Mansky PJ. Safety and efficacy of acupuncture in children: a review of the evidence. J Pediatr Hematol Oncol. 2008;30(6):431-42. doi: 10.1097/MPH.0b013e318165b2cc. [PubMed: 18525459]. [PubMed Central: PMC2518962].

67. Food. Complementary and alternative medicine products and their regulation by the Food and Drug Administration. Food and Drug Administration. 2006. Available from: https://www.fda.gov/regulatoryinformation/search-fda-guidance-documents/complementaryand-alternative-medicine-products-and-their-regulation-foodand-drug-administration.

68. Lauche R, Cramer H, Choi KE, Rampp T, Saha FJ, Dobos GJ, et al. The influence of a series of five dry cupping treatments on pain and mechanical thresholds in patients with chronic non-specific neck paina randomised controlled pilot study. BMC Complement Altern Med. 2011;11:63. doi: 10.1186/1472-6882-11-63. [PubMed: 21843336]. [PubMed Central: PMC3224248].

69. Chi LM, Lin LM, Chen CL, Wang SF, Lai HL, Peng TC. The Effectiveness of Cupping Therapy on Relieving Chronic Neck and Shoulder Pain: A Randomized Controlled Trial. Evid Based Complement Alternat Med. 2016;2016:7358918. doi: 10.1155/2016/7358918. [PubMed: 27073404]. [PubMed Central: PMC4814666].

70. Nasb M, Qun X, Ruckmal Withanage C, Lingfeng X, Hong C. Dry Cupping, Ischemic Compression, or Their Combination for the Treatment of Trigger Points: A Pilot Randomized Trial. J Altern Complement Med. 2020;26(1):44-50. doi: 10.1089/acm.2019.0231. [PubMed: 31580695]. [PubMed Central: PMC6983744].

71. Trofa DP, Obana KK, Herndon CL, Noticewala MS, Parisien RL, Popkin CA, et al. The Evidence for Common Nonsurgical Modalities in Sports Medicine, Part 2: Cupping and Blood Flow Restriction. J Am Acad Orthop Surg Glob Res Rev. 2020;4(1). e1900105. doi: 10.5435/JAAOSGlobalD-19-00105. [PubMed: 32672728]. [PubMed Central: PMC7028774].

72. Gerber LH, Shah J, Rosenberger W, Armstrong K, Turo D, Otto P, et al. Dry Needling Alters Trigger Points in the Upper Trapezius Muscle and Reduces Pain in Subjects With Chronic Myofascial Pain. PM R. 2015;7(7):711-8. doi: 10.1016/j.pmrj.2015.01.020. [PubMed: 25661462]. [PubMed Central: PMC4508220].

73. Cerezo-Tellez E, Torres-Lacomba M, Fuentes-Gallardo I, Perez-Munoz M, Mayoral-Del-Moral O, Lluch-Girbes E, et al. Effectiveness of dry needling for chronic nonspecific neck pain: a randomized, single-blinded, clinical trial. Pain. 2016;157(9):1905-17. doi: 10.1097/j.pain.0000000000000591. [PubMed: 27537209].

74. De Meulemeester KE, Castelein B, Coppieters I, Barbe T, Cools A, Cagnie B. Comparing Trigger Point Dry Needling and Manual Pressure Technique for the Management of Myofascial Neck/Shoulder Pain: A Randomized Clinical Trial. J Manipulative Physiol Ther. 2017;40(1):11-20. doi: 10.1016/j.jmpt.2016.10.008. [PubMed: 28017188].

75. Furlan AD, Yazdi F, Tsertsvadze A, Gross A, Van Tulder M, Santaguida L, et al. Complementary and alternative therapies for back pain II. Evid Rep Technol Assess (Full Rep). 2010;(194):1-764. [PubMed: 23126534]. [PubMed Central: PMC4781408].

76. Fu LM, Li JT, Wu WS. Randomized controlled trials of acupuncture for neck pain: systematic review and meta-analysis. J Altern Complement Med. 2009;15(2):133-45. doi: 10.1089/acm.2008.0135. [PubMed: 
19216662].

77. Que Q, Ye X, Su Q, Weng Y, Chu J, Mei L, et al. Effectiveness of acupuncture intervention for neck pain caused by cervical spondylosis: study protocol for a randomized controlled trial. Trials. 2013;14:186. doi: 10.1186/1745-6215-14-186. [PubMed: 23800342]. [PubMed Central: PMC3700747].

78. Gu CL, Yan Y, Zhang D, Li P. An evaluation of the effectiveness of acupuncture with seven acupoint-penetrating needles on cervical spondylosis. J Pain Res. 2019;12:1441-5. doi: 10.2147/JPR.S199798. [PubMed: 31123418]. [PubMed Central: PMC6511242].

79. Li W, Cong W, Yan C, Zhang R, Gao Y, Ma Y. [Clinical observation of fast acupuncture for cervical type of cervical spondylosis]. Zhongguo Zhen Jiu. 2017;37(9):951-4. Chinese. doi: 10.13703/j.0255-2930.2017.09.010. [PubMed: 29354916].

80. Ho LF, Lin ZX, Leung AWN, Chen L, Zhang H, Ng BFL, et al. Efficacy of abdominal acupuncture for neck pain: A randomized controlled trial. PLoS One. 2017;12(7). e0181360. doi: 10.1371/journal.pone.0181360. [PubMed: 28715459]. [PubMed Central: PMC5513533].

81. Calamita SAP, Biasotto-Gonzalez DA, De Melo NC, Fumagalli MA, Amorim CF, de Paula Gomes CAF, et al. Immediate Effect of Acupuncture on Electromyographic Activity of the Upper Trapezius Muscle and Pain in Patients With Nonspecific Neck Pain: A Randomized, Single-Blinded, Sham-Controlled, Crossover Study. J Manipulative Physiol Ther. 2018;41(3):208-17. doi: 10.1016/j.jmpt.2017.09.006. [PubMed: 29549891].

82. Barreto TW, Svec JH. Chronic Neck Pain: Nonpharmacologic Treatment. Am Fam Physician. 2019;100(3):180-2. [PubMed: 31361100].

83. Seo SY, Lee KB, Shin JS, Lee J, Kim MR, Ha IH, et al. Effectiveness of Acupuncture and Electroacupuncture for Chronic Neck Pain: A Systematic Review and Meta-Analysis. Am J Chin Med. 2017;45(8):1573-95. doi: 10.1142/S0192415X17500859. [PubMed: 29121797].

84. Eslamian F, Jahanjoo F, Dolatkhah N, Pishgahi A, Pirani A. Relative Effectiveness of Electroacupuncture and Biofeedback in the Treatment of Neck and Upper Back Myofascial Pain: A Randomized Clinical Trial. Arch Phys Med Rehabil. 2020;101(5):770-80. doi: 10.1016/j.apmr.2019.12.009. [PubMed:31954696].

85. Kim E, Kim YS, Kim YI, Jeon JH, Yoo HR, Park YC, et al. Effectiveness and Safety of Polydioxanone Thread-Embedding Acupuncture as an Adjunctive Therapy for Patients with Chronic Nonspecific Neck Pain: A Randomized Controlled Trial.JAltern Complement Med.2019;25(4):41726. doi: 10.1089/acm.2018.0228. [PubMed: 30523703].

86. Farag AM, Malacarne A, Pagni SE, Maloney GE. The effectiveness of acupuncture in the management of persistent regional myofascial head and neck pain: A systematic review and meta-analysis. Complement Ther Med. 2020;49:102297. doi: 10.1016/j.ctim.2019.102297. [PubMed: 32147064].

87. Yang $\mathrm{Y}$, Yan $\mathrm{X}$, Deng $\mathrm{H}$, Zeng D, Huang J, Fu W, et al. The efficacy of traditional acupuncture on patients with chronic neck pain study protocol of a randomized controlled trial. Trials. 2017;18(1):312. doi: 10.1186/s13063-017-2009-1. [PubMed: 28693563]. [PubMed Central: PMC5504675].

88. Liang ZH, Di Z, Jiang S, Xu SJ, Zhu XP, Fu WB, et al. The optimized acupuncture treatment for neck pain caused by cervical spondylosis: a study protocol of a multicentre randomized controlled trial. Trials. 2012;13:107. doi:10.1186/1745-6215-13-107. [PubMed: 22776567]. [PubMed Central: PMC3460740]

89. Sun M, Geng G, Chen J, Ma X, Yan M, Liu X, et al. Acupuncture for chronic neck pain with sensitive points: study protocol for a multicentre randomised controlled trial.BMJOpen.2019;9(7). e026904.doi: 10.1136/bmjopen-2018-026904. [PubMed: 31366643]. [PubMed Central: PMC6678032].

90. Sun M, Tao S, Geng G, Peng J, Ma X, Yan M, et al. Identification of the optimal points for the acupuncture treatment of neck pain in China: protocol for a multicenter, matched, case-control study. BMJ Open.2019;9(8).e029194.doi:10.1136/bmjopen-2019-029194. [PubMed 31439605]. [PubMed Central: PMC6707690].

91. Zuo G, Gao TC, Xue BH, Gu CC, Yan YT, Zhang YW, et al. Assessment of the efficacy of acupuncture and chiropractic on treating Cervical spondylosis radiculopathy: A systematic review and meta-analysis. Medicine (Baltimore). 2019;98(48). e17974. doi 10.1097/MD.0000000000017974. [PubMed: 31770206]. [PubMed Central: PMC6890346].

92. Pan H, Jin R, Li M, Liu Z, Xie Q, Wang P. The Effectiveness of Acupuncture for Osteoporosis: A Systematic Review and Meta-Analysis. Am J Chin Med. 2018;46(3):489-513. doi: 10.1142/S0192415X18500258. [PubMed: 29614884].

93. Chen D, Ni XX, Wang LJ, Zeng Q, Xie Y], Zhao L. [Literature quality analysis of RCTs regarding acupuncture for chronic neck pain]. Zhongguo Zhen Jiu. 2019;39(8):889-95. doi:10.13703/j.0255-2930.2019.08.025. [PubMed: 31397138].

94. Lorenc A, Feder G, MacPherson H, Little P, Mercer SW, Sharp D. Scoping review of systematic reviews of complementary medicine for musculoskeletal and mental health conditions. BMJ Open. 2018;8(10). e020222. doi: 10.1136/bmjopen-2017-020222. [PubMed: 30327397] [PubMed Central: PMC6196876]. 


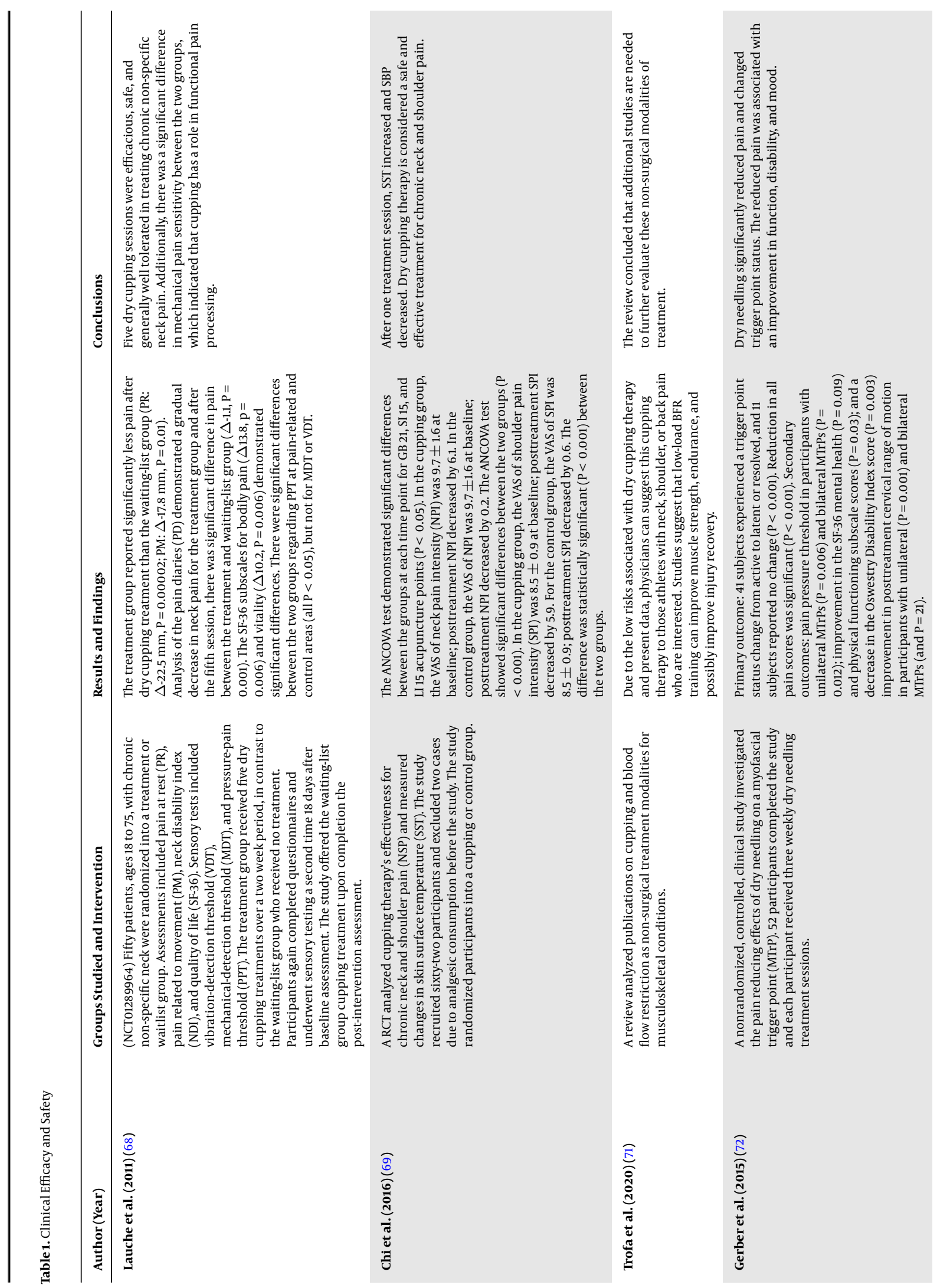



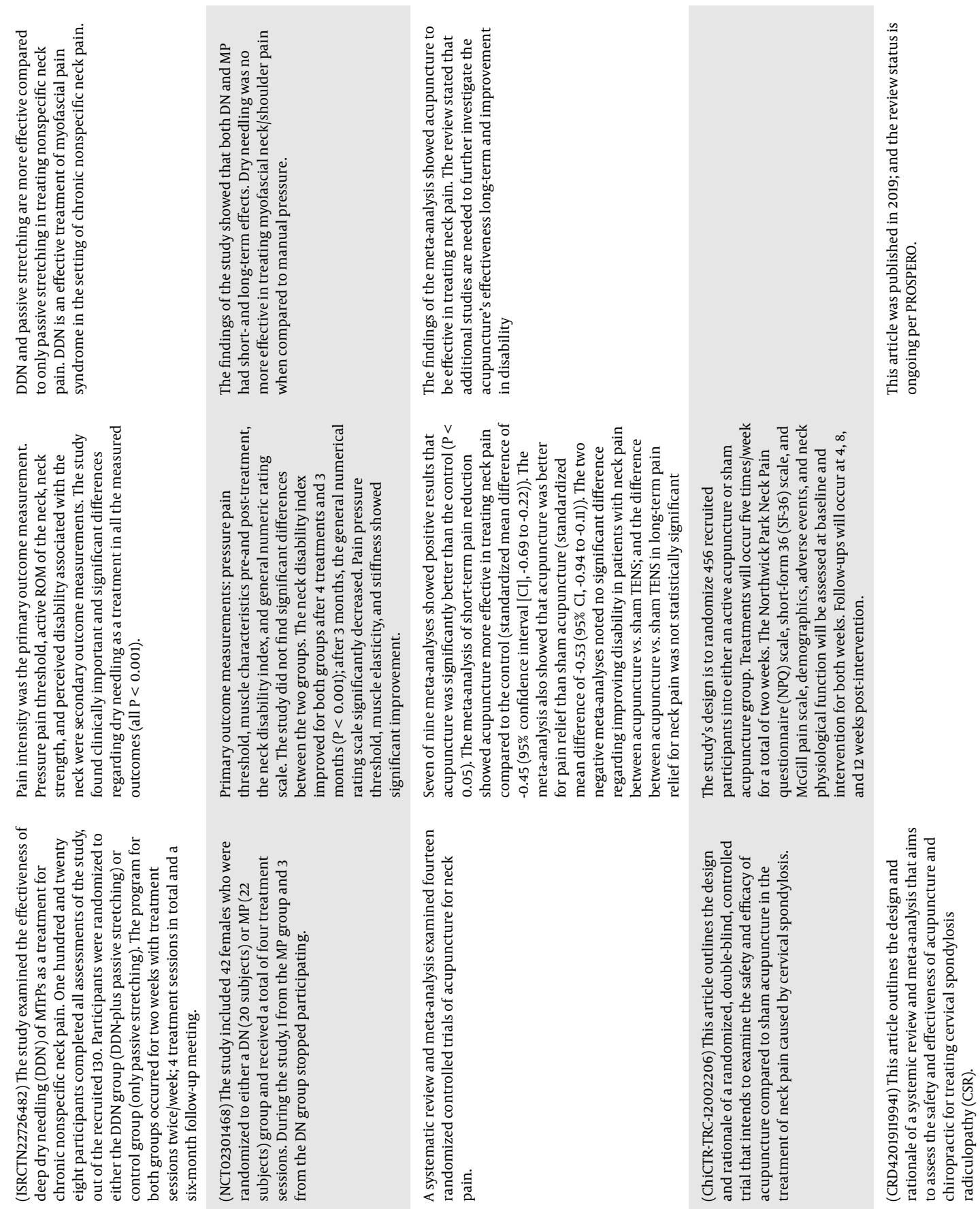

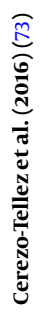
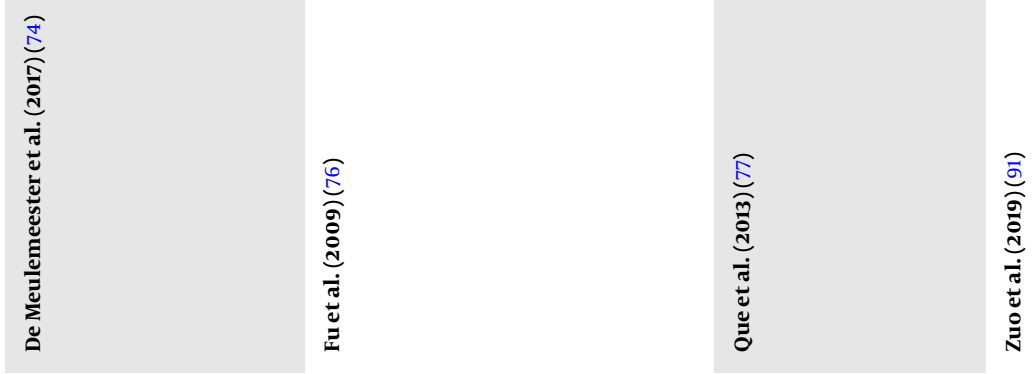


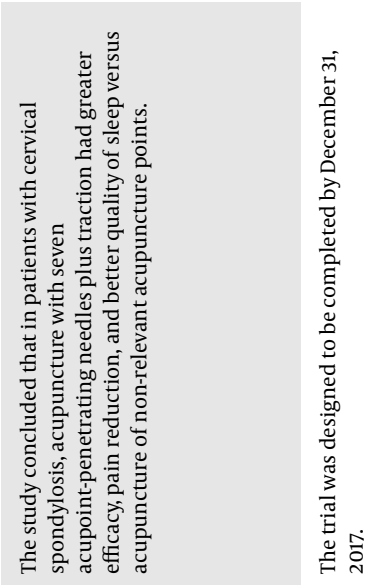

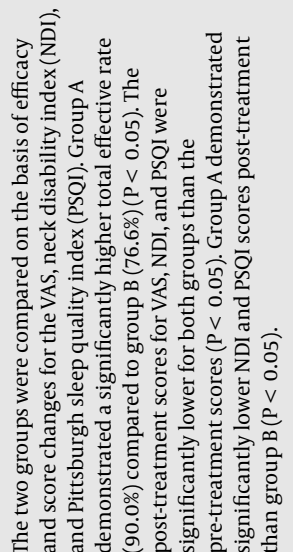

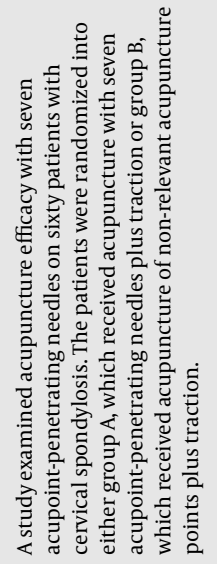

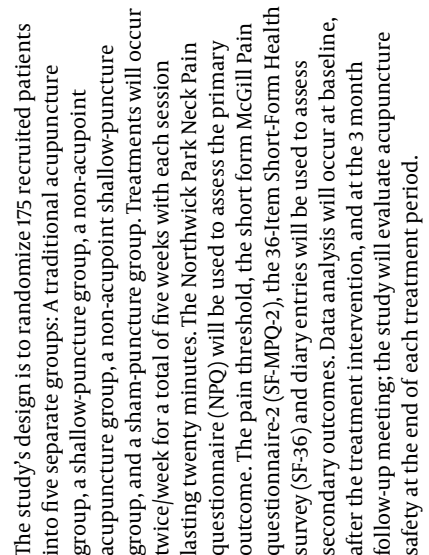
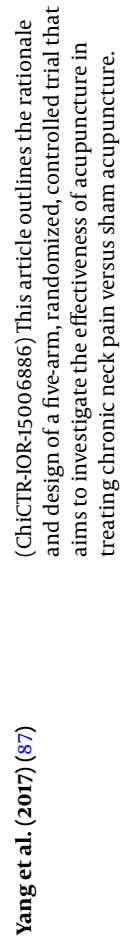
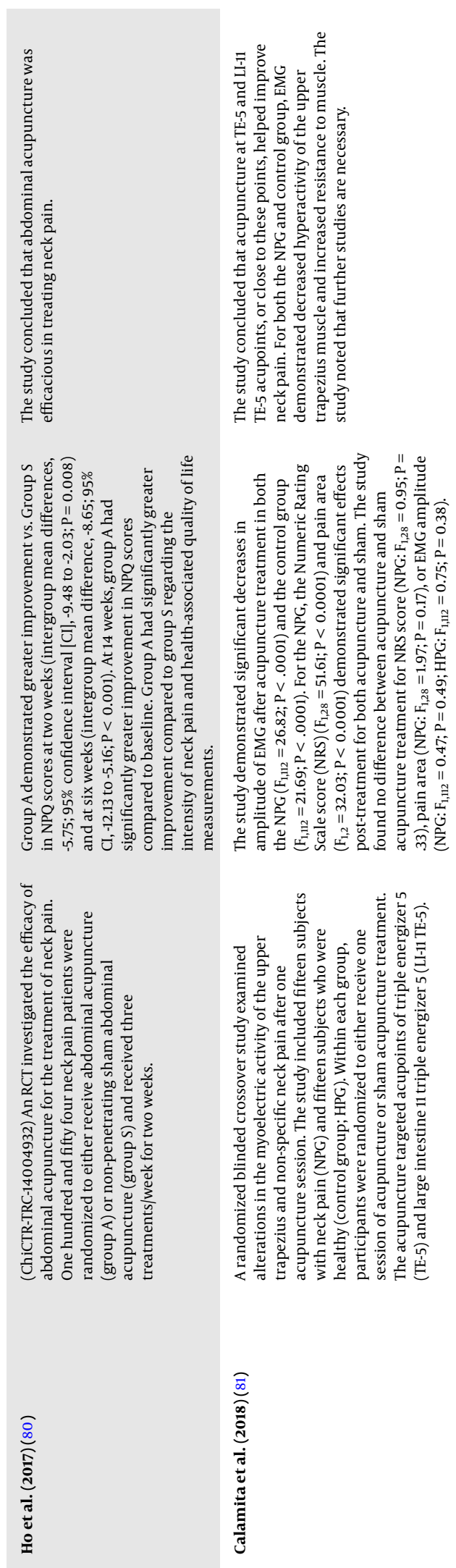

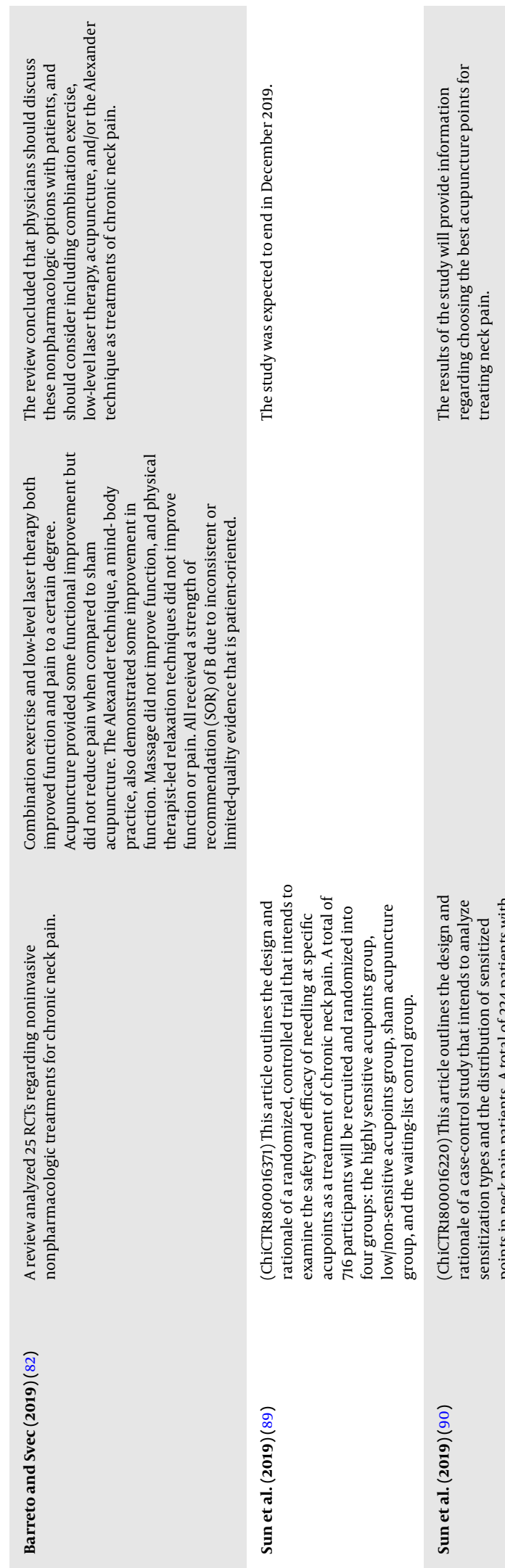
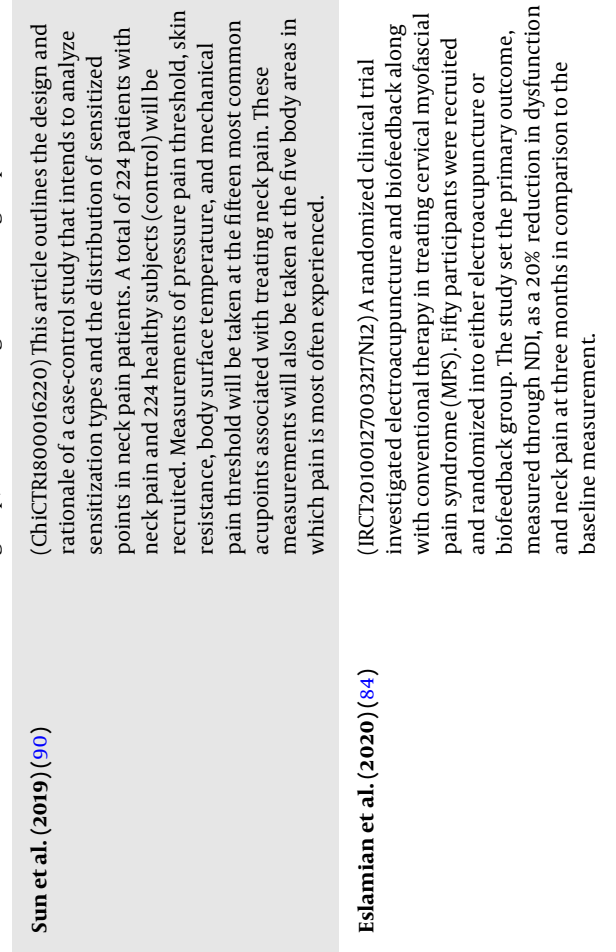
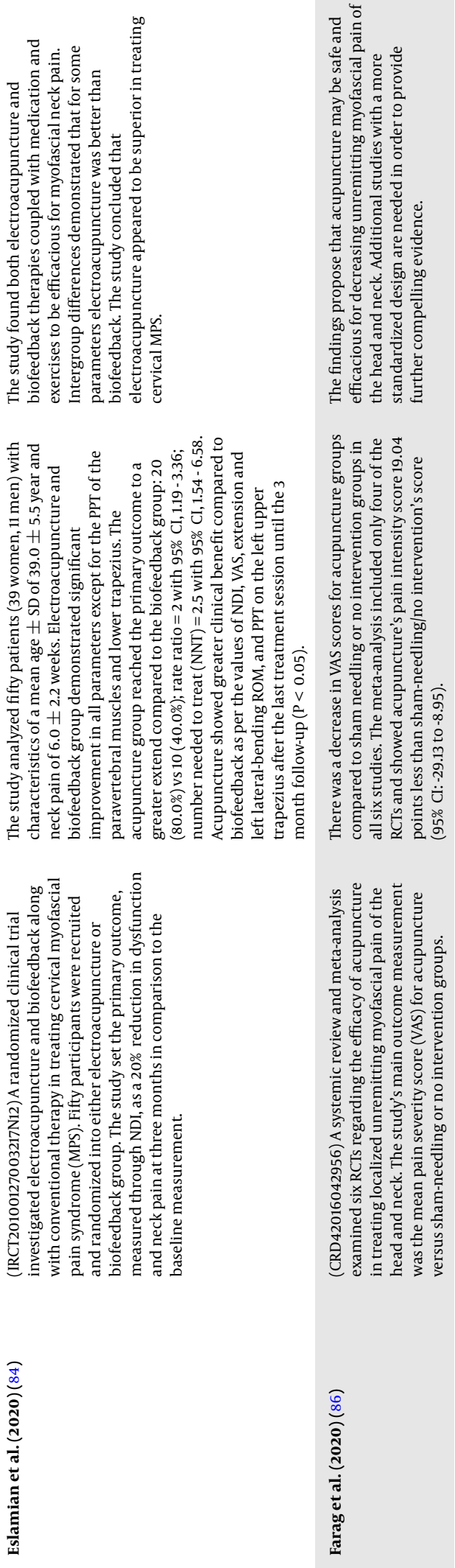


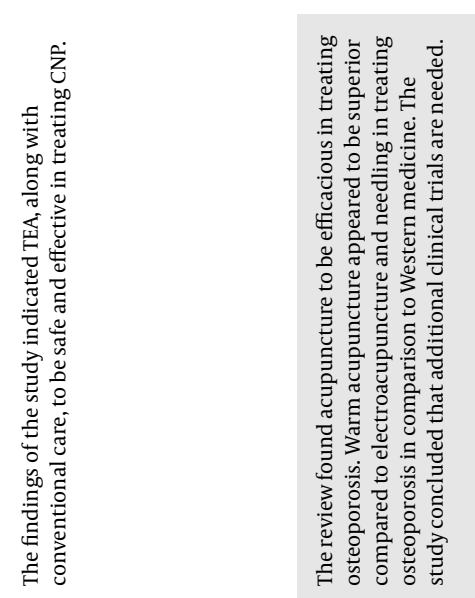

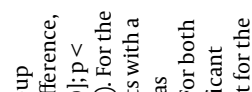

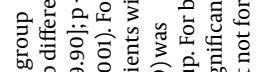

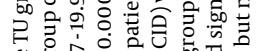

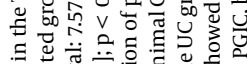

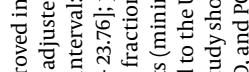

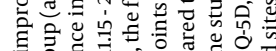

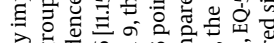

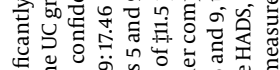

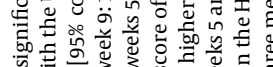

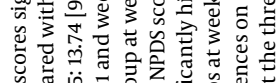

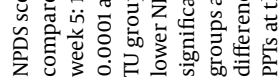

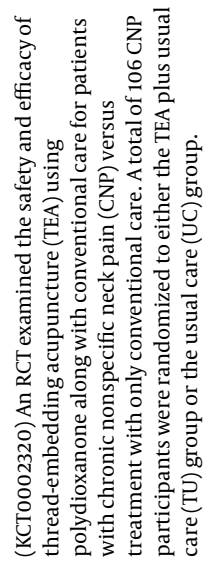

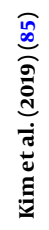

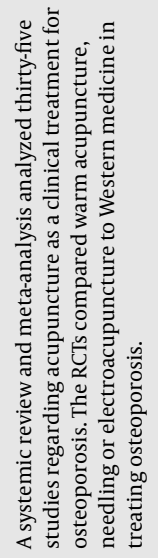

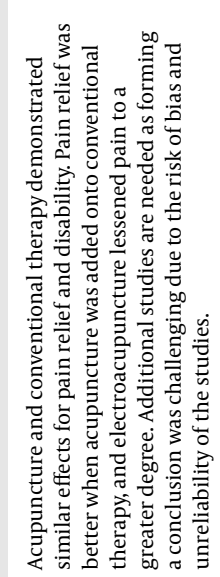
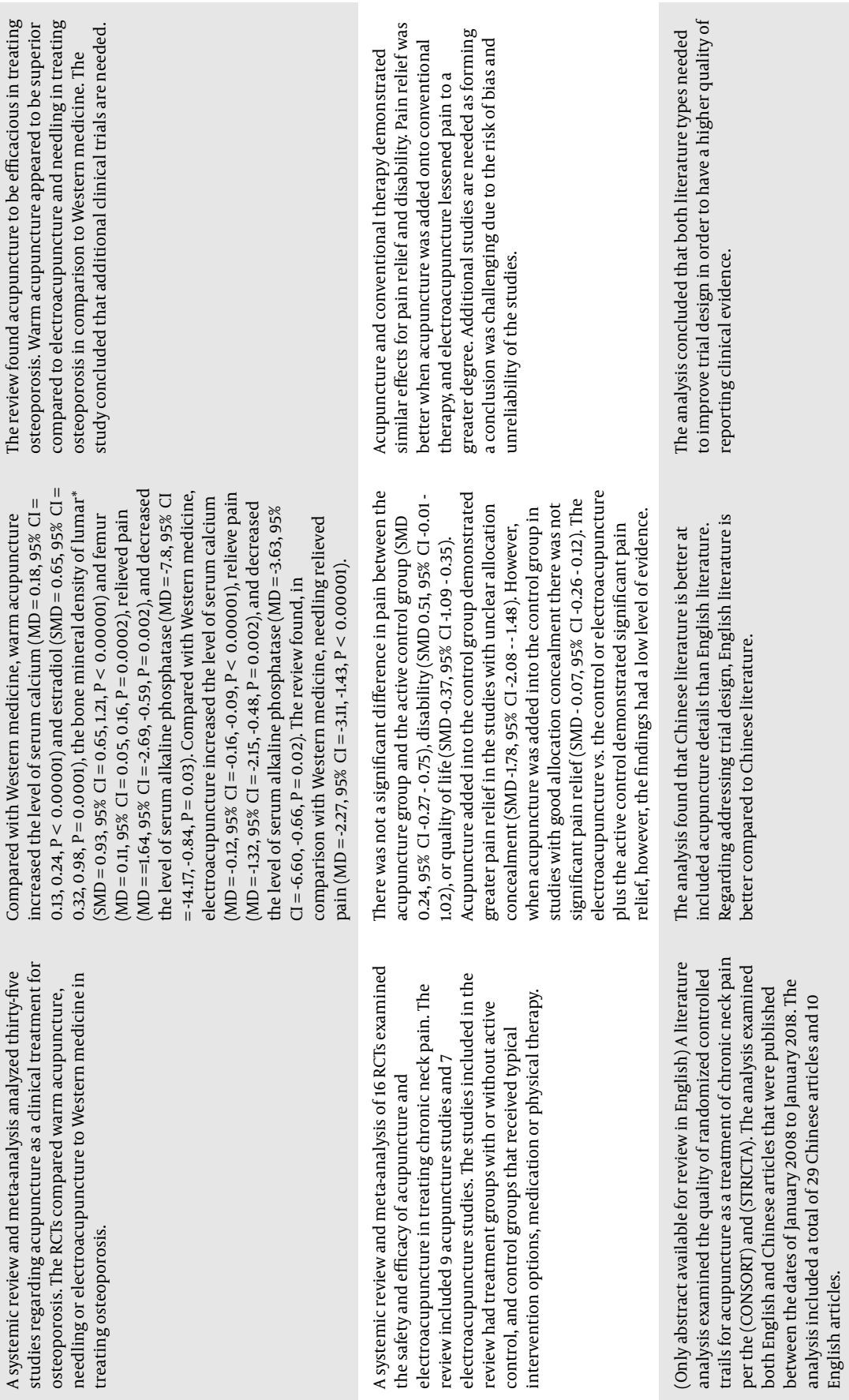

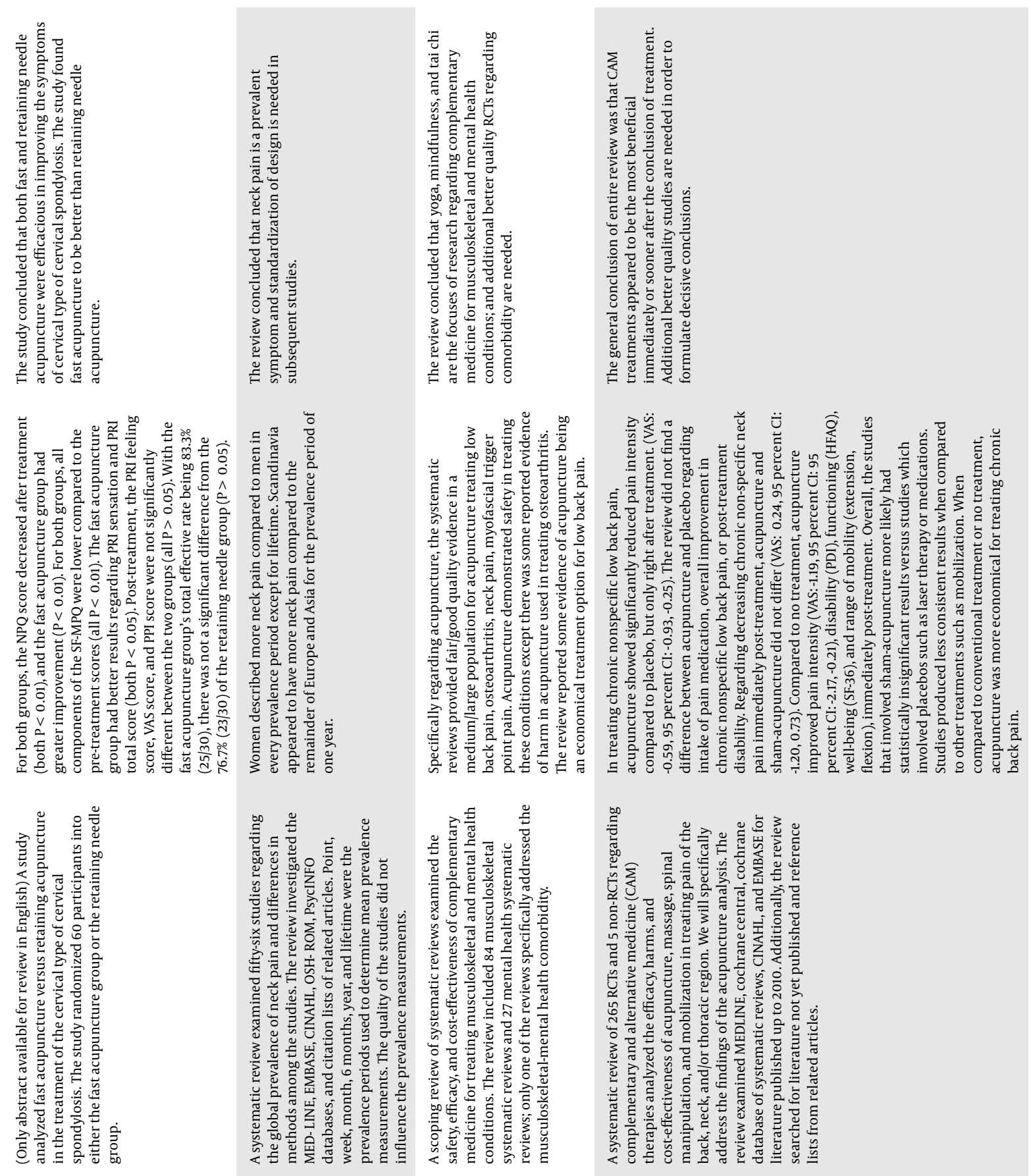

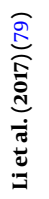

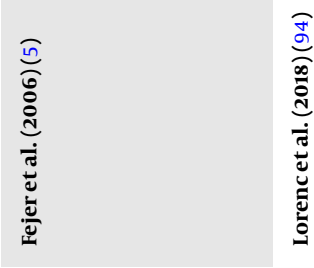

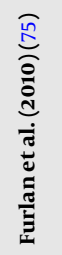



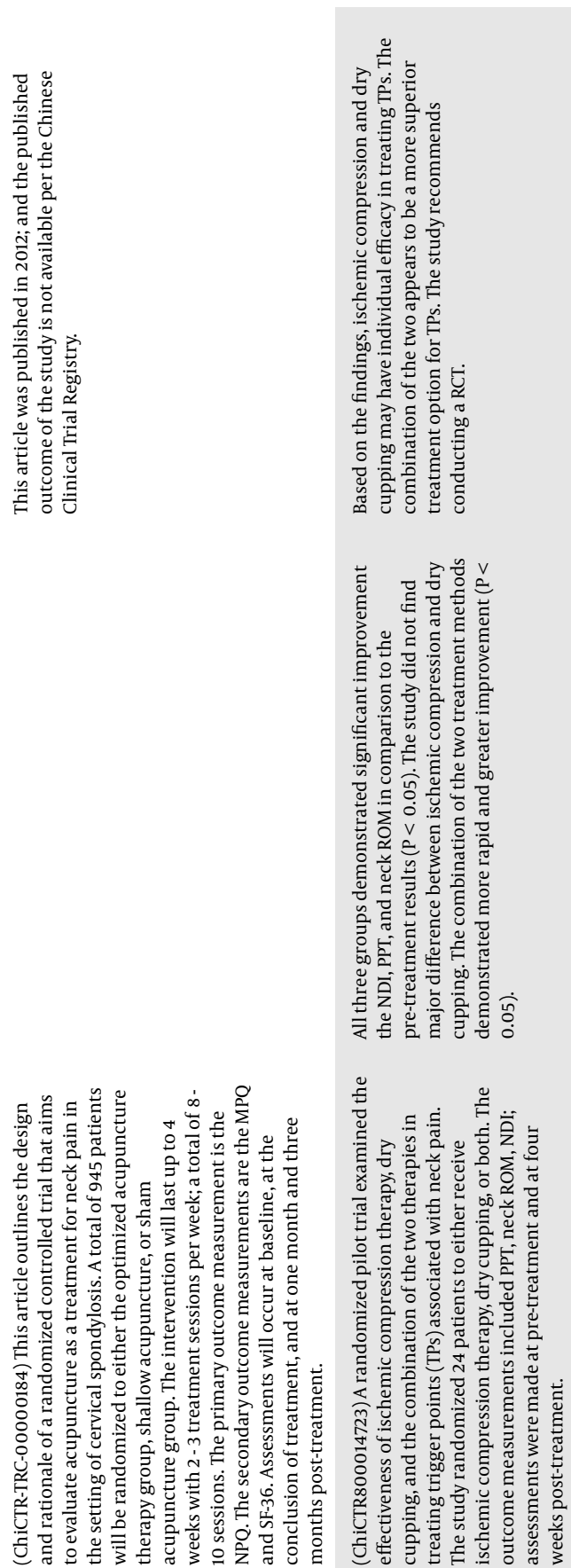

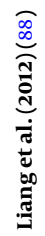

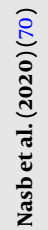

